\title{
Parlamentarische Demokratie versus Volksgesetzgebung. Der Streit um ein neues Wahlrecht in Hamburg
}

\section{Frank Decker}

In Hamburg spielt sich zur Zeit ein Lehrstück in Sachen Demokratie ab, das man vielleicht als Lokalposse abtun könnte, wenn es nicht für das Verhältnis der Bürger zur Politik und für die Akzeptanz des parteiendemokratischen parlamentarischen Systems ganz grundsätzliche Fragen aufwerfen würde. In diesem Stück geht es zum einen um den Stellenwert der so genannten Volksgesetzgebung, zum anderen um eine Reform des Wahlrechts. ${ }^{1}$ Gerungen wird also um die Ausgestaltung der beiden institutionellen Wege, auf denen das Volk - wie es das Grundgesetz in Art. 20 Abs. 2 ausdrückt - die Staatsgewalt ausübt.

An beiden Fronten hat es in der Bundesrepublik auf kommunaler und Länderebene in den letzten eineinhalb Jahrzehnten bemerkenswerte Änderungen gegeben, durch die die demokratischen Mitwirkungsmöglichkeiten der Bürger vermehrt wurden. ${ }^{2}$ Auf der Landesebene manifestierte sich der Demokratisierungsschub in erster Linie in der Einführung beziehungsweise im Ausbau der direktdemokratischen Verfahren. Diese waren zwar in den meisten Ländern von Hause aus vorgesehen, spielten im Verfassungsleben aber keine nennenswerte Rolle. Das Zusammentreffen mehrerer Faktoren führte dazu, dass sich das ab Ende der achtziger Jahre änderte: Als wichtigste Hintergrundvariable muss hier das gewachsene Bedürfnis nach politischer Partizipation gesehen werden. Günstige Gelegenheiten für eine Reform entstanden zudem durch die Notwendigkeit, die vor dem Grundgesetz erlassenen Landesverfassungen zu modernisieren, was durch politische Ereignisse mancherorts noch verstärkt wurde. So war es kein Zufall, dass die Erneuerung ausgerechnet in Schleswig-Holstein ihren Ausgang nahm, das Ende der achtziger Jahre die Barschel-Affäre zu verkraften hatte. Nachhaltige Rückwirkung auf den Reformprozess entfaltete schließlich die Verfassungsgebung in den neuen Ländern. Auch wenn die zum Teil radikalen Vorstellungen, die man in den Beratungen des Runden Tisches entwickelt hatte, im parlamentarischen Entscheidungsverfahren weitgehend abgeschliffen wurden, setzten die Verfassungen in Ostdeutschland einen neuen demokratischen Trend, der den Ruf nach Veränderung auch in der alten Bundesrepublik lauter werden ließ.

Wesentlich bescheidener und weniger innovativ waren die Reformen im Bereich des Wahlrechts. Auch die neuen Länder orientierten sich hier sämtlich am personalisierten Verhältniswahlrecht des Bundes mit Erst- und Zweitstimme, starren Listen und FünfProzent-Klausel, während die Änderungen in der Altbundesrepublik auf die nachträgliche Übernahme des Zwei-Stimmen-Systems beschränkt blieben (so in Hessen, Niedersachsen und Rheinland-Pfalz). Zu einer Abkehr von den starren Listen mochte sich bis

1 Tobias Kublmann, „Kleinere Korrekturen“, Die Zeit Nr. 25 vom 14. Juni 2006, S. 15.

2 Frank Decker, Die Regierungssysteme in den Ländern, in: ders. (Hrsg.), Föderalismus an der Wegscheide? Optionen und Perspektiven einer Reform der bundesstaatlichen Ordnung, Wiesbaden 2004, S. $169-201$. 
zum Erfolg der Volksinitiative in Hamburg kein Bundesland durchringen. Allein Bayern eröffnet dem Wähler im Landtagswahlrecht die Chance, mit der Zweitstimme unter mehreren Bewerbern einer Partei auszuwählen. ${ }^{3}$ Eine weitere Reform, die auf den ersten Blick als Entdemokratisierung gedeutet werden könnte, bei Berücksichtigung der erweiterten plebiszitären Beteiligungsmöglichkeiten aber durchaus gerechtfertigt erscheint, betrifft die Dauer der Wahlperiode. Diese hat man nach dem Vorbild des Saarlandes inzwischen in allen Ländern außer in Hamburg und Bremen auf fünf Jahre verlängert.

Zum eigentlichen Motor der Reform avancierten in den neunziger Jahren die Kommunen. Die Demokratisierungsbemühungen richteten sich hier nicht nur auf die Verfahren der direkten Demokratie (Bürgerbegehren und Bürgerentscheid) ${ }^{4}$, deren Einführung in den meisten Fällen parallel zu derjenigen auf Landesebene erfolgte. Sie betrafen zugleich das Wahlrecht, wobei das Moment der Personenwahl deutlich gestärkt wurde. Dazu dienten zum einen der Wegfall oder die Absenkung der Fünf-Prozent-Hürde, zum anderen die Einführung von Elementen des Kumulierens und Panaschierens. Letztere sind mittlerweile in den meisten Kommunalverfassungen verbreitet. Neben den drei Stadtstaaten, von denen aufgrund ihrer Bezirksstruktur aber nur Hamburg und Berlin wirklich miteinander vergleichbar sind, halten lediglich Nordrhein-Westfalen, das Saarland und Schleswig-Holstein weiterhin am Prinzip starrer Listen fest. Ähnlich sieht es bei den Sperrklauseln aus, auf die heute zwölf Länder ganz verzichten. In einigen Fällen (Berlin, Mecklenburg-Vorpommern und Nordrhein-Westfalen) bedurfte es dabei allerdings der Nachhilfe durch die Landesverfassungsgerichte, um die Parteien zur Korrektur der bestehenden Regelungen zu zwingen.

Dass sich die Demokratisierung der Wahlsysteme am süddeutschen Vorbild orientierte, war kein Zufall, ging sie doch mit einer grundlegenden Umgestaltung auch der kommunalen Regierungsformen einher. Diese führte in den neunziger Jahren in sämtlichen Bundesländern zur Übernahme der so genannten Süddeutschen Ratsverfassung, die bis dahin nur in Bayern und Baden-Württemberg gegolten hatte. Die Einführung der Direktwahl war gleichbedeutend mit dem Übergang von der parlamentarischen zur präsidentiellen Regierungsform. Statt sie legitimatorisch miteinander zu verschränken, werden Rat und Bürgermeister nun getrennt voneinander bestellt, was beide Organe unabhängiger voneinander macht und den Bürgern neue Partizipationschancen eröffnet. Einige Länder gingen sogar soweit, als logisches Pendant zur Direktwahl auch die Möglichkeit einer vorzeitigen Abwahl des Bürgermeisters durch das Volk (den so genannten recall) in den Verfassungen zu verankern. ${ }^{5}$ Diese Möglichkeit gab es in Bayern und Baden-Württemberg nicht, und sie ist dort auch nachträglich nicht eingeführt worden.

3 Vgl. zu dieser Möglichkeit und deren Nutzung durch die Wähler auch Thorsten Faas / Harald Schoen, Reihenfolgeeffekte bei der bayerischen Landtagswahl 2003: Die Ersten werden die Ersten sein, in: ZParl, 36. Jg. (2005), H. 1, S. $100 \mathrm{ff}$.

4 Vgl. dazu auch den Beitrag von Jens Kösters in diesem Heft der ZParl.

5 In acht Bundesländern ist die Abberufung nur auf Initiative der Vertretungskörperschaft möglich (Hessen, Mecklenburg-Vorpommern, Niedersachsen, Nordrhein-Westfalen, RheinlandPfalz, Saarland, Sachsen-Anhalt, Thüringen), in drei Ländern darüber hinaus auch auf Initiative der Bürger selbst (Brandenburg, Sachsen, Schleswig-Holstein). Vgl. Jan Witte, Der kommunale „Recall“ in Deutschland - erste Anwendungserfahrungen, in: ZParl, 32. Jg. (2001), H. 1, S. $57 \mathrm{ff}$. 
Anders als das parlamentarische System ist die präsidentielle Regierungsform nicht auf organisatorisch und ideologisch fest gefügte Parteien angewiesen, die dauerhaft stabile Abstimmungsmehrheiten hervorbringen. Konstellationen des divided government und wechselnde Koalitionen sind in den Gemeinden gang und gäbe und stellen dort aufgrund des sach- und verwaltungsbezogenen Charakters der Aufgaben kein ernstes Problem dar. Dass es „keinen christlich-demokratischen Straßenbau und keine sozialdemokratische Wasserwirtschaft" gibt, so Wilhelm Hennis ${ }^{6}$, bleibt in der Tendenz richtig. Daher können es sich die Kommunen auch leisten, beim Wahlrecht großzügig zu verfahren und die Stabilitätsorientierung des parlamentarischen Systems zugunsten einer stärkeren demokratischen Offenheit zurückzustellen, die auch Einzelbewerbern und den Kandidaten kleinerer Parteien die Chance gibt, in die Vertretungskörperschaften zu gelangen.

Der Vergleich zwischen der staatlichen Politik des Bundes und der Länder mit der Kommunalpolitik ist gerade im Blick auf die Stadtstaaten instruktiv, die ja beide Bereiche miteinander verbinden. Der Doppelcharakter zeigt sich dabei nicht nur im Vorhandensein einer eigenständigen kommunalen (bezirklichen) Ebene unterhalb der staatlichen. Sie kommt vielmehr darin zum Ausdruck, dass auch auf der staatlichen Ebene Aufgaben wahrzunehmen sind, die in den Flächenländern in die Zuständigkeit der Kommunen fallen. Daher liegt die Frage nahe, ob sich die Stadtstaaten nicht auch in institutioneller Hinsicht - bei der Gestaltung ihrer Regierungssysteme - eher an den kommunalen als an den Landesverfassungen orientieren müssten.

Letzteres scheint umso plausibler, als sich die Landespolitik in der Bundesrepublik auch sonst dem Charakter der Kommunalpolitik immer stärker angenähert hat. Der Grund dafür liegt in der Auszehrung der Länderkompetenzen durch den Bund. Die Hauptaufgaben der Länder im deutschen Föderalismus liegen im Verwaltungsbereich und in ihrer Mitwirkung an der Bundesgesetzgebung. Diese Aufgaben werden freilich überwiegend oder ausschließlich von den Exekutiven wahrgenommen, was zur verbreiteten Klage über den Funktionsverlust der Landesparlamente geführt hat. Sieht man vom Bereich Schule und Hochschule ab, gibt es kaum noch einen Politikbereich, in dem die Länder über eigene legislative Gestaltungsmöglichkeiten verfügen. Um nicht ganz überflüssig zu werden, sind die Landesparlamente in der Vergangenheit deshalb zunehmend auf Felder ausgewichen, deren Regelung man genauso gut den Kommunen überlassen könnte.

\section{Hamburg: Vom Schlusslicht zum Vorreiter der Demokratisierung}

Angesichts der Affinität der Landespolitik im Allgemeinen und der Politik der Stadtstaaten im Besonderen zur kommunalen Ebene ist es verblüffend, dass die institutionellen Reformbemühungen an der Freien und Hansestadt Hamburg lange Zeit spurlos vorübergingen. Hamburg war das letzte Bundesland, das 1996 die direktdemokratischen Verfahren in seine Verfassung einfügte und damit den vorläufigen Schlussstein unter den plebis-

6 Wilhelm Hennis, Parlamentarische Opposition und Industriegesellschaft. Zur Lage des parlamentarischen Regierungssystems, in: Gesellschaft - Staat - Erziehung, 1. Jg. (1956), S. 205 222, wiederabgedruckt in: ders., Regieren im modernen Staat. Politikwissenschaftliche Abhandlungen I, Tübingen 1999, S. 1 - 23. 
zitären Reformprozess setzte. ${ }^{7}$ Das Instrument galt zunächst nur auf der Landesebene und bewegte sich hier, was die Anwenderfreundlichkeit angeht, im Mittelfeld aller Bundesländer. Auf der kommunalen Ebene wurden die Plebiszite erst 1998 eingeführt. ${ }^{8}$ Dem war ein vom Verein „Mehr Demokratie“ angestrengtes Volksbegehren vorausgegangen, das die Bürgerschaft zwang, die Sache selbst in die Hand zu nehmen und eine Konkurrenzvorlage zu unterbreiten. Obwohl sich die Initiative mit ihrem eigenen Entwurf gegen diese Vorlage nicht durchsetzen konnte, gingen auch die dort enthaltenen Regelungen so weit, dass sie Hamburg bundesweit zu einem Vorreiter der kommunalen Direktdemokratie machten. ${ }^{9}$

Beim Wahlrecht blieb die Hansestadt demgegenüber weiter das demokratische Schlusslicht. ${ }^{10}$ Sowohl auf der Landesebene als auch auf der kommunalen Bezirksebene konnte man hier nur eine Liste wählen, deren personelle Zusammensetzung ausschließlich von den Parteien bestimmt wurde. Die Geltung derselben Regeln und die Zeitgleichheit beider Abstimmungen führten zudem dazu, dass die Ergebnisse der Bürgerschaftswahl mit jenen der sieben Bezirksversammlungswahlen fast immer übereinstimmten, die letzteren also funktional betrachtet im Grunde überflüssig waren. Die Rückschrittlichkeit des Hamburger Wahlsystems wäre gemessen an der Situation in den anderen Ländern bereits dann bemerkenswert gewesen, wenn es sich bei der Hansestadt ausschließlich um ein Bundesland und nicht zugleich um eine Kommune handeln würde. Auf der Landesebene gibt es nämlich heute mit dem Saarland und Bremen nur noch zwei Länder, die an einem Einstimmenwahlrecht mit starren Listen festhalten. Im Unterschied zu Hamburg wird ein Großteil der Mandate aber auch hier zunächst über die Wahlkreislisten besetzt, bevor die Landesliste zum Zuge kommt. In der Hansestadt konnte man sich demgegenüber noch nicht einmal auf die Schaffung von Wahlkreisen verständigen, um ein Mindestmaß an Bürgernähe herzustellen.

Dass der Demokratisierungsprozess dann doch in Gang kam, lag nicht an der Einsicht der Parteien. Es verdankte sich vielmehr den neu geschaffenen Institutionen der direkten Demokratie, die von den Bürgern als Hebel benutzt werden konnten, um die demokratischen Reformen auch gegen den Willen des Parlaments herbeizuführen. Indem das plebiszitäre Instrument auf sich selbst angewendet wurde, gelang es nicht nur, die Direktdemokratie auf die Bezirke auszudehnen, sondern auch ihre Anwendbarkeit auf der Landesebene deutlich zu verbessern. Ein von „Mehr Demokratie“ zu diesem Zweck eingeleitetes Volksbegehren für eine Verfassungsänderung verfehlte zwar 1998 das erforderliche Quorum. Die klare Mehrheit für den Entwurf wurde aber von der Bürgerschaft als

7 Dies gilt allerdings nicht in Bezug auf die kommunale Ebene. Hier blieb das Schlusslicht bis vor kurzem Berlin, das die plebiszitären Verfahren lediglich auf der Landesebene eingeführt hatte und sich erst im Jahre 2005 dazu durchrang, Bürgerbegehren und Bürgerentscheid auch in den Bezirken zu ermöglichen.

8 Zum Vergleich des Einsatzes direktdemokratischer Instrumente in den Bundesländern vgl. Ralph Kampwirth, Der ernüchterte Souverän. Bilanz und Perspektiven der direkten Demokratie in den 16 Bundesländern und auf Kommunalebene, in: ZParl, 34. Jg. (2003), H. 4, S. $657 \mathrm{ff}$.

9 Andreas Fraude, Direkte Demokratie in Hamburg, in: Andreas Kost (Hrsg.), Direkte Demokratie in den Ländern, Wiesbaden 2005, S. $113-132$.

10 Vgl. zum Wahlrecht allgemein auch Paul Tiefenbach, Kumulieren, Panaschieren, Mehrmandatswahlkreise - mehr Demokratie beim Wahlrecht?, in: ZParl, 37. Jg. (2006), H. 1, S. 115 ff. 
Aufforderung verstanden, die Volksgesetzgebung von sich aus zu novellieren. Die 2001 beschlossene Senkung der Quoren für Volksbegehren und Volksentscheid trug den Vorschlägen der Initiative Rechnung. ${ }^{11}$ Mit ihr rückte Hamburg, was die direktdemokratische Praxis angeht, im Bundesländervergleich weit nach oben.

Dass unter den erleichterten Anwendungsbedingungen auch das Wahlrecht irgendwann in das Visier der Initiatoren geraten würde, hätten die Parteien eigentlich vorausahnen müssen. Anders als die in der Verfassung verankerte Volksgesetzgebung kann das Wahlrecht ja einfachgesetzlich geändert werden, womit es auch den direktdemokratischen Verfahren leichter zugänglich ist. Union und SPD mussten aber auch dafür zum Jagen getragen werden. Zu einer Reform waren sie erst bereit, als eine neuerliche Volksinitiative ihren Entwurf für ein neues Wahlrecht bereits auf den Weg gebracht hatte. Die Konkurrenzvorlage der beiden Volksparteien sah für Hamburg die Übernahme des Bundestagswahlrechts vor. Dies stellte im Vergleich zum Status quo zweifellos einen Fortschritt dar, reichte aber nicht aus, um den sehr viel weitergehenden Vorschlag der Initiative abzuwehren, der in der Schlussabstimmung im Juni 2004 von zwei Dritteln der Wähler unterstützt wurde. Allerdings gelang es hierbei nur knapp (und dank der zeitlichen Kopplung an die Europawahl), das notwendige Zustimmungsquorum von 20 Prozent zu überspringen.

Das neue Gesetz sah für Hamburg die Schaffung von 17 Mehrpersonenwahlkreisen vor, in denen 71 der insgesamt 121 Bürgerschaftskandidaten gewählt werden sollten. ${ }^{12}$ Durch die Mehrpersonenwahlkreise wollte man eine größere Proportionalität erreichen, indem nun auch kleinere Parteien und Einzelbewerber die Chance erhalten würden, ein Mandat zu erringen. Dem Wähler wurden für den Wahlkreis fünf Stimmen zugestanden - mit der Möglichkeit, diese auf unterschiedliche Parteien und / oder Kandidaten zu verteilen (Kumulieren und Panaschieren). Die restlichen 50 Mandate wären über Listen verteilt worden. Auch hier hätte der Wähler fünf Stimmen beliebig vergeben können. Die Gesamtmandatsverteilung hätte sich allein nach den Listenstimmen gerichtet. Dasselbe System wäre bei den Wahlen zu den sieben Bezirksversammlungen angewandt worden, allerdings ohne die Fünf-Prozent-Klausel und die bisherige zeitliche Kopplung an die Bürgerschaftswahl. Stattdessen sollten die Bezirkswahlen zusammen mit den alle fünf Jahre stattfindenden Europawahlen abgehalten werden.

Misst man die demokratische Qualität des Wahlrechts an den personellen Auswahlmöglichkeiten für den Wähler, so hätte das von der Volksinitiative durchgesetzte Gesetz Hamburg in der Landespolitik vom letzten auf den ersten Platz und in der Kommunalpolitik vom letzten auf einen der vordersten Plätze aller Wahlsysteme in der Bundesrepublik katapultiert. Unter Reformgesichtspunkten wäre das gewiss zu begrüßen gewesen. In der Radikalität steckte jedoch zugleich ein Problem, da die Neuerungen den Bürgern

11 Zur Problematik von Zustimmungsquoren vgl. Thorsten Hüller, Herrschaft des Quorums? Ein Vorschlag zur Lösung eines Problems direkter Demokratie, in: ZParl, 37. Jg. (2006), H. 4, S. $823 \mathrm{ff}$

12 HmbGVBl. Nr. 33 vom 14. Juli 2004, S. 313 - 320. Eine bürgerfreundliche Darstellung, die von der Landeszentrale für politische Bildung der Hansestadt vertrieben worden ist und nach der Abwicklung des Wahlrechts wohl aus dem Verkehr gezogen werden wird, findet sich bei Katrin Eggers, Das neue Wahlrecht in Hamburg. Ein Leitfaden für alle Wählerinnen und Wähler, Hamburg 2005. 
wie auch den Parteien ein hohes Maß an Lernbereitschaft abverlangten: Die Bürger hätten sich auf ein komplizierteres System umstellen und die Parteien akzeptieren müssen, dass sie ihre bisherige vollständige Kontrolle über die Rekrutierung der Mandatsträger verlieren. Zumindest der CDU war dieses Maß offenbar zuviel. Ein langsameres Tempo der Demokratisierung wäre hier womöglich sinnvoller gewesen, weil es die Parteien dann leichter gehabt hätten, die Reformen in die eigenen Reihen hinein zu vermitteln. Dazu hätten sich Union und SPD aber aufraffen müssen, das Wahlsystem von sich aus zu demokratisieren, wozu sie in Hamburg weder willens noch in der Lage waren. Erst der drohende Erfolg der Volksinitiative hat sie - viel zu spät - veranlasst, den Reformpfad zu betreten. Insofern tragen die beiden Volksparteien die Hauptverantwortung für die schwierige Situation, die durch den radikalreformerischen Ansatz der Initiative eingetreten ist.

\section{Die von der CDU durchgesetzten Änderungen am Wahlrecht}

Die CDU hat ihre Ankündigung wahrgemacht, das vom Volk beschlossene Wahlrecht noch in der laufenden Legislaturperiode zu „kippen“ - also bevor das Gesetz überhaupt zum ersten Mal angewendet wird. Mit ihrer absoluten Mehrheit setzte sie am 11. Oktober 2006 in der Bürgerschaft ein neues Wahlgesetz durch, das den alten Entwurf in wesentlichen Punkten abändert. ${ }^{13}$ Obwohl es in der Partei durchaus Stimmen gab, die von dem Vorhaben abrieten und an der Basis wenig Sympathie für die Änderungspläne herrschte, stimmten bei der Schlussabstimmung 62 von 63 CDU-Abgeordneten dafür. Schon im September 2005 hatte eine deutliche Mehrheit des CDU-Landesausschusses den Weg für die Rückgängigmachung des Volksbeschlusses freigemacht.

Es ist nicht ohne Ironie, dass die für die Ausarbeitung der Änderungsvorschläge eingesetzte Kommission ausgerechnet vom inzwischen verstorbenen ehemaligen CDU-Landesvorsitzenden Jürgen Echternach geleitet wurde, mit dessen Namen sich eine der unrühmlichsten Episoden der Hamburger Nachkriegspolitik verbindet. Echternach hatte die Hamburger CDU mit einem Kreis von Getreuen seit den siebziger Jahren autoritär geführt und sich dabei über die verfassungs- und satzungsrechtlich geschützten Prinzipien der innerparteilichen Demokratie immer wieder hinweggesetzt. ${ }^{14}$ Dies führte nicht nur zu heftigen Auseinandersetzungen innerhalb des Landesverbandes, sondern hatte auch juristische Konsequenzen, die in der Annullierung einer kompletten Bürgerschaftswahl durch das Landesverfassungsgericht im Jahre 1993 ihren Höhepunkt fanden. ${ }^{15}$ Die der Union von höchstrichterlicher Seite bescheinigten Demokratieverstöße wurden vom Wähler bei der anschließenden Neuwahl rigoros abgestraft ${ }^{16}$ und wirkten als Katalysator

13 Vgl. CDU zieht Änderungen durch, in: Hamburger Abendblatt vom 12. Oktober 2006.

14 Vgl. Martin Müller / Katrin Börsting, Kandidatenaufstellung und innerparteiliche Willensbildung am Beispiel der Hamburger CDU, in: Jürgen Hartmann / Uwe Thaysen (Hrsg.), Pluralismus und Parlamentarismus in Theorie und Praxis. Winfried Steffani zum 65. Geburtstag, Opladen 1992, S. $137-173$.

15 Vgl. Jörn Ipsen, Kandidatenaufstellung, innerparteiliche Demokratie und Wahlprüfungsrecht. Anmerkung zum Urteil des Hamburgischen Verfassungsgerichts vom 4. Mai 1993, in: ZParl, 25. Jg. (1994), H. 2, S. 235 ff.

16 Vgl. dazu auch Ursula Feist / Hans-Jürgen Hoffmann, Die Hamburger Bürgerschaftswahl vom 
für den Erfolg der „Statt-Partei“ - einer von CDU-Dissidenten gegründeten bürgerlichen Protestbewegung. ${ }^{17}$

Obwohl diese Ereignisse noch gar nicht so lange zurückliegen, scheinen sie in der CDU heute keine größeren Skrupel mehr auszulösen. Die Motive, die der Rücknahme des volksbeschlossenen Wahlrechts zugrunde liegen, sind dieselben wie damals. Es geht um die Zurückgewinnung der vollständigen Kontrolle über den Rekrutierungsprozess und damit um die Absicherung der Macht der parteilichen Führungsgruppen. Zwischen diesen und der mittleren Funktionärsschicht der Partei besteht dabei eine weitgehende Interessenidentität. Die Führung vertraut auf die Loyalität der Funktionäre, und die Funktionäre erhalten dafür im Gegenzug ein bestimmtes Maß an Planungssicherheit, was ihre eigene Parteikarriere angeht. Letzteres wäre nicht mehr gewährleistet, wenn bei der Kandidatennominierung Außenseiter an den „Platzhirschen“ ungehindert vorbeiziehen könnten.

Bisher verfügten die Parteien in Hamburg bei der Auswahl der Kandidaten über ein faktisches Monopol. Dieses wäre durch das vom Volk beschlossene Wahlrecht in mehrerlei Hinsicht geknackt worden. Zum einen hätten parteiunabhängige Einzelbewerber eine größere Chance erhalten, gewählt zu werden. Des Weiteren hätten die Wähler wesentlich stärker als bisher mitentschieden, welche Kandidaten ihrer Parteien ein Mandat erhalten und darüber hinaus sogar Einfluss auf das Personaltableau der von ihnen nicht primär unterstützten Parteien nehmen können. Dies hätte zugleich die Parteibasis gegenüber der Führung gestärkt, da die einzelnen Parteigliederungen infolge der größeren Auswahlmöglichkeiten zusätzliche Kandidaten hätten aufstellen müssen. Last but not least wären die Chancen für kleinere, bisher nicht im Parlament vertretene Gruppierungen gestiegen, Mandate zu erringen. Bezogen auf die Listenstimme gilt das insbesondere für die Bezirksversammlungswahlen, bei denen es keine Fünf-Prozent-Hürde mehr gegeben hätte.

Im Wesentlichen sind es drei Änderungen, mit denen die CDU die intendierte Stärkung der personellen Auswahlmöglichkeiten rückgängig gemacht und den Status quo ante einer weitgehenden Kontrolle der Parteien über den Rekrutierungsprozess wieder hergestellt hat. ${ }^{18}$ Erstens wird die Möglichkeit des Kumulierens und Panaschierens bei der Zweitstimme zugunsten der bisherigen starren Liste mit nur einer Parteistimme abgeschafft. Zweitens hat man die Änderung der Reihenfolge auf der Wahlkreisliste dadurch erschwert, dass eine bestimmte Quote von Persönlichkeitsstimmen erfüllt sein muss, um bei der Mandatsvergabe Berücksichtigung zu finden. Der hierfür vorgesehene Wert von 30 Prozent der Wahlzahl ${ }^{19}$ dürfte nach den Erfahrungen anderer Kommunalwahlsysteme eine kaum überwindliche Hürde darstellen. Drittens werden bei den Bezirksversammlungswahlen die Fünf-Prozent-Klausel sowie die heutige Koppelung an die Bürgerschafts-

19. September 1993: Rundumangriff auf die Etablierten, in: ZParl, 25. Jg. (1994), H. 2, S. $217 \mathrm{ff}$.

17 Vgl. Frank Decker, Die Hamburger STATT Partei. Ursprünge und Entwicklung einer bürgerlichen Wählerbewegung, in: Jahrbuch für Politik, 4. Jg. (1994), 2. Halbband, S. 249 - 294.

18 Der Entwurf des Wahlrechts-Änderungsgesetzes wurde von der CDU am 19. Juni 2006 in die Bürgerschaft eingebracht (Bürgerschaft der Freien und Hansestadt Hamburg, Drucksache 18 / 4339).

19 Die Wahlzahl wird ermittelt, indem man die Gesamtzahl der abgegebenen gültigen Stimmen in einem Wahlkreis durch die Zahl der zu vergebenden Mandate (drei, vier oder fünf) dividiert. 
wahlen wieder eingeführt. ${ }^{20}$ Daneben gibt es eine Reihe von anderen Änderungen (etwa die Nachrückerregelung), die in dieselbe Richtung zielen, in ihrer Auswirkung aber geringer zu veranschlagen sind.

Begründet werden die Änderungen sowohl mit Praktikabilitätserwägungen als auch mit den vermeintlich negativen Auswirkungen des volksbeschlossenen Wahlrechts auf die Qualität des politischen Personals. Was ersteres betrifft, weist die Union insbesondere auf die drohende Überforderung des Wählers hin, wenn dieser zwischen einer großen Zahl von Kandidaten auswählen müsse, von denen er nur einen Bruchteil persönlich oder dem Namen nach kenne. Darüber hinaus sei das Wahlverfahren zu kompliziert, weshalb mit einem Anstieg der ungültigen Stimmen zu rechnen sei. Dass diese Argumente wenig stichhaltig sind, zeigen die Erfahrungen aus anderen Bundesländern, wo die Wähler bei Kommunalwahlen zum Teil noch sehr viel mehr Stimmen zur Verfügung haben als sie unter dem volksbeschlossenen Wahlrecht in Hamburg gehabt hätten. Die Zahl der ungültigen Stimmen nimmt zwar im Vergleich zur reinen Listenwahl zu, doch ist der Wert längst nicht so dramatisch, dass er die positiven Demokratieeffekte aufwiegen könnte. ${ }^{21}$

Noch weniger überzeugend sind die mit Blick auf die Qualität des politischen Personals erhobenen Bedenken. Hier herrscht die Sorge, dass ein Wahlrecht mit zu großen personellen Auswahlmöglichkeiten zu einer Deprofessionalisierung des Parlamentsbetriebs führen könne, die die Handlungsfähigkeit der Bürgerschaft insgesamt gefährde. Während Politparvenüs, Populisten und Querulanten vom Zufallsfaktor einer Personenwahl profitieren würden, hätten die geduldig arbeitenden, politikerfahrenen Fachleute

20 Eine weitere interessante Regelung, mit der die Union zugleich wahlrechtliches Neuland betritt, liegt in der Einführung einer so genannten Mehrheitssicherungsklausel ( $\$ 5$ Abs. 4 Satz 3 und 4 des Gesetzes über die Wahl zur Hamburgischen Bürgerschaft). Damit soll sichergestellt werden, dass eine Partei, die bei der Listenwahl die absolute Mehrheit der Stimmen erzielt, in jedem Fall auch die absolute Mehrheit der Sitze in der Bürgerschaft erhält. Ob die Einfügung einer solchen mehrheitswahlrechtlichen Komponente in das bestehende Verhältniswahlsystem verfassungsgemäß ist, wird von Kritikern bezweifelt. Für die Verfassungsgemäßheit könnte sprechen, dass ein mögliches Auseinanderfallen von elektoraler und parlamentarischer Mehrheit infolge von Überhangmandaten in einigen Länderwahlrechten schon heute durch Ausgleichsmandate verhindert werden kann. Anders als die „Berlusconi-Klausel“ in Italien soll die neue Vorschrift der Mehrheit ja keinen zusätzlichen Bonus einräumen, sondern diese Mehrheit überhaupt erst herstellen. Die Bedenken gegen die Mehrheitsklausel sind denn auch eher verfassungspolitischer Natur. Das Argument der Regierungsstabilität, das die CDU für die Einführung der Klausel vorbringt, mag auf der Bundesebene seine Berechtigung haben. Auf der Ebene des Landes oder der Kommunen greift es aber - wie gesehen - nur bedingt. Dies gilt umso mehr, als die Mehrheitsregierung in einem Bundesland schon heute über eine starke Position verfügt. Anders als die Regierung im Bund muss sie sich zum Beispiel nicht mit einer in großen Teilen der Gesetzgebung gleichberechtigt mitwirkenden Zweiten Kammer auseinandersetzen. Ein stärkerer Konsenszwang auf der parlamentarischen Ebene könnte von daher durchaus sinnvoll sein, zumal er auch die Position der Bürgerschaft im Ganzen gegenüber dem Senat aufwerten würde.

21 Vgl. Paul Tiefenbach, a.a.O., S. 118. In den Ländern, deren Kommunalwahlsysteme Kumulieren und Panaschieren zulassen, schwankte die Quote der ungültigen Stimmen bei den zuletzt stattgefundenen Kommunalwahlen zwischen 2,4 (Niedersachsen 2006) und 5,3 Prozent (Sachsen-Anhalt 2004). Der Durchschnittswert aller Länder lag mit 4,2 Prozent genau doppelt so hoch wie der entsprechende Wert von 2,1 für die Landtagswahlen (Zweitstimme). 
der Fraktionen das Nachsehen. Auch an diesem Punkt belegen Untersuchungen ${ }^{22}$ aus den Ländern, deren Kommunalwahlsysteme Kumulieren und Panaschieren erlauben, dass der Anteil der Abgeordneten, die darauf rechnen könnten, an den besser platzierten Kandidaten vorbei in die Parlamente zu ziehen, vermutlich nicht mehr als ein Viertel betragen würde. Die Parteien hätten also weiter die Möglichkeit, ihre Experten und Spitzenleute auf den Listen abzusichern. Um die letzteren bräuchten sie ohnehin nicht zu fürchten, da sich die Wähler bei einer Persönlichkeitswahl erfahrungsgemäß stark von der Bekanntheit eines Kandidaten leiten lassen.

Das Qualitätsargument steht noch in anderer Hinsicht auf schwachen Füßen. Ein Nachteil des volksbeschlossenen Wahlrechts mag darin gelegen haben, dass es die Möglichkeit erschwert hätte, über die Zusammensetzung der Listen auch Minderheiten und Quereinsteiger angemessen zu berücksichtigen (zum Beispiel durch Proporz- oder Quotenregelungen). Gerade von dieser Möglichkeit haben die etablierten Parteien in der Vergangenheit jedoch keinen überzeugenden Gebrauch gemacht. Stattdessen begünstigte das überkommene Listenwahlsystem die Rekrutierung eines Abgeordnetentypus, für dessen Karriereweg sich in der Publizistik der wenig schmeichelhafte Begriff der „Ochsentour" durchgesetzt hat. Dies birgt, wie man weiß, Probleme, da es bestimmte politiknahe Berufsgruppen einseitig privilegiert. ${ }^{23}$ Vertreter aus anderen gesellschaftlichen Bereichen wie Wirtschaft, Kultur oder Wissenschaft haben es demgegenüber weiterhin schwer, ihren Weg in die Parlamente zu finden. Ein offeneres Wahlsystem könnte hier vielleicht Abhilfe schaffen.

\section{Zum Verhältnis von parlamentarischer und Volksgesetzgebung}

Obwohl die offiziell vorgebrachten Argumente gegen das volksbeschlossene Wahlrecht wie gesehen - die wahre Motiv- und Interessenlage der CDU nur zum Teil widerspiegeln, sind sie doch diskussionswürdig. Tatsächlich wäre das von der Partei jetzt beschlossene System ein Fortschritt gewesen, wenn man es bereits vor zehn Jahren eingeführt hätte. So aber bleibt die Auseinandersetzung um das Für und Wider der Änderungsvorschläge müßig, da sie das eigentliche Problem verfehlt. Dieses liegt in der offensichtlichen Missachtung des 2004 getroffenen Volksentscheids.

Die besondere Brisanz des Vorgangs liegt darin, dass die regierende CDU-Mehrheit in dieser Beziehung ein „Wiederholungstäter" ist. Der Rücknahme des volksbeschlossenen Wahlgesetzes waren in derselben Legislaturperiode bereits zwei andere Fälle vorausgegangen, in denen sich die Bürgerschaft dem durch Abstimmung geäußerten Volkswillen ausdrücklich oder der Tendenz nach widersetzt hatte. So hielt das mit klarer Mehrheit

22 Vgl. Paul Tiefenbach, a.a.O., S. 117, und Klaus Hofmann, Wahlrecht und Gewählte. Die Auswirkungen des Wahlrechts auf die Zusammensetzung der Städte- und Gemeinderäte, Köln 2006, wo der höchste Wert der Mandatsrelevanz für das hessische Kommunalwahlrecht ermittelt wird (20 Prozent).

23 Vgl. Elmar Wiesendahl, Elitenrekrutierung in der Parteiendemokratie. Wer sind die Besten und setzen sie sich in den Parteien durch?, in: Oscar W. Gabriel / Beate Neuss / Günther Rüther (Hrsg.), Konjunktur der Köpfe? Eliten in der modernen Wissensgesellschaft, Düsseldorf 2004, S. $124-141$. 
(von 76,8 Prozent) getroffene Votum der Bürger für einen Verbleib der Landeskrankenhäuser im Staatsbesitz, das im Februar 2004 zeitgleich mit der Bürgerschaftswahl erfolgte, den Senat nicht davon ab, die beabsichtigte Veräußerung dennoch vorzunehmen. ${ }^{24}$ Das von den Initiatoren daraufhin angerufene Hamburgische Verfassungsgericht bestätigte dieses Vorgehen als verfassungsgemäß. Zum einen habe es sich bei dem Volksentscheid nur um eine Aufforderung an Senat und Bürgerschaft gehandelt, der keine rechtsverbindliche Wirkung zukomme. Zum anderen wäre das Parlament auch bei einem qua Volksentscheid ergangenen Gesetzesbeschluss befugt gewesen, diesen abzuändern. Der Grundsatz der Organtreue gebiete lediglich, die Auffassung der anderen Staatsorgane bei der eigenen Entscheidung angemessen zu berücksichtigen, was das Gericht im vorliegenden Fall als gegeben ansah. ${ }^{25}$

Weniger erfolgreich war die Regierungspartei bei ihrem Versuch, die Regeln der Volksgesetzgebung selbst zu verändern. Obwohl sie die Senkung der Quoren für Volksbegehren und Volksentscheid in der Bürgerschaft seinerzeit mit getragen hatte, nahm die CDU die empfindlichen Abstimmungsniederlagen bei der Krankenhausprivatisierung und beim Wahlrecht jetzt zum Anlass, die Anwendbarkeit der direktdemokratischen Verfahren an zwei Stellen zu erschweren. Die Volksentscheide sollten künftig nicht mehr gleichzeitig mit einer regulären Wahl abgehalten werden und die Bürger sich nur noch auf einer Behörde in die Unterstützerliste für ein Volksbegehren eintragen dürfen. Letzteres wurde vom Verfassungsgericht mit der Einschränkung gebilligt, dass die Neuregelung nicht für bereits zustande gekommene Volksinitiativen gelte. Der beabsichtigten Entkoppelung der Abstimmungen von den Wahlen schoben die Richter hingegen einen Riegel vor, da die Hamburgische Verfassung die gleichzeitige Durchführung ausdrücklich zulasse. $^{26}$

Die Hamburger Oppositionsparteien SPD und GAL haben die Anrufung des Verfassungsgerichts gegen die Wahlrechtsnovelle bereits angekündigt. $\mathrm{Ob}$ sie am Ende Recht bekommen werden, ist insofern fraglich, als das Gericht in seinem Urteil zum Krankenhausverkauf an der Berechtigung des parlamentarischen Gesetzgebers, ein volksbeschlossenes Gesetz zu ändern, keinen Zweifel gelassen hat. Hamburg fügt sich hier, was die Rechtsprechung angeht, ganz in die Praxis der anderen deutschen Landesverfassungen, wo ein besonderer Bestandsschutz für volksbeschlossene Gesetze ausnahmslos nicht vorgesehen ist. Diese können also von den Parlamenten (wie auch vom Verfassungsgericht) genauso korrigiert oder rückgängig gemacht werden, wie das Volk umgekehrt die Möglichkeit hat, die nachträgliche Änderung oder Aufhebung eines Parlamentsgesetzes zu begehren. Dabei sind jenseits der einzuhaltenden Verfahrensvorschriften weder besondere Fristen zu beachten, noch bedarf es einer „wesentlichen Veränderung der Rechts- oder Sachlage“, um den Gesetzgeber zum Eingreifen zu legitimieren. ${ }^{27} \mathrm{Ob}$ die Abgeordneten

24 Vgl. zum Volksentscheid und zur Wahl der Hamburger Bürgerschaft Patrick Horst, Die Neuwahl zur Hamburger Bürgerschaft vom 29. Februar 2004: die Hanseaten und Angela Merkel finden einen politischen Hoffnungsträger, in: ZParl, 35. Jg. (2004), H. 2, S. 252 ff., S. 259.

25 HVerfG 6/04 = u.a. DÖV 2005, S. 252 ff.; DVBl., S. 438 ff. mit Anmerkung Klaas Engelken, S. 415 ff.; NVwZ, S. 685 ff.; NordÖR, S. 109 ff. mit Anmerkung Hans Peter Bull, S. 99 ff.

26 HVerfG 2/05 = u.a. DÖV 2006, S. 1001; NordÖR, S. 348; DVBl., S. 1590 ff.

27 HVerfG 6/04 (Fn. 25); kritisch zur wissenschaftlichen Diskussion dieser Frage nur: Otmar Jung, Die rebellierende Vertretung, in: Andreas Bovenschulte / Henning Grub / Franziska A. Löhr 
von ihrer Befugnis Gebrauch machen, ist mithin allein eine Frage der politischen Opportunität. Der Respekt vor dem Volksvotum und die Angst vor einer möglichen Sanktionierung durch den Wähler stehen hier gegen das eigene Machtkalkül oder die Überzeugung, etwas Richtiges zu tun. Letzteres galt und gilt aus Sicht der CDU sicherlich für ihren Entschluss, sich dem von den Bürgern verlangten Verbleib der Krankenhäuser im Staatsbetrieb zu verweigern. Die Änderungen des Volksgesetzgebungsgesetzes und des Wahlrechts dürften demgegenüber primär von dem Bedürfnis diktiert worden sein, einen unbequemen Vetospieler im politischen Prozess zu entmachten.

Wie ist die nachträgliche Änderung oder Aufhebung eines volksbeschlossenen Gesetzes unter Demokratiegesichtspunkten zu bewerten? Die verfassungsrechtlichen Maßstäbe geben zur Beantwortung dieser Frage nicht sehr viel her. Wenn parlamentarisch und plebiszitär zustande gekommene Gesetze rechtlich betrachtet gleichrangig sind, heißt das schließlich nicht, dass eine vollständige Symmetrie der beiden Verfahrenstypen auch in politischer Hinsicht besteht. Da Volksentscheidungen wesentlich seltener vorkommen als Parlamentsentscheidungen und sich dann zumeist auf Fragen beziehen, die von den Bürgern als besonders wichtig empfunden werden, könnte man ihnen im Gegenteil sogar eine höherwertige Legitimität zuschreiben, die sich auch in einer entsprechenden Bindungswirkung niederschlagen muss. Politiker gefallen sich, wie man weiß, vorzugsweise an Wahlabenden darin, den Wähler als „Souverän“ zu titulieren. Dies kommt einer Verhöhnung gleich, wenn man im Gegenzug nicht bereit ist, die Entscheidungen des nämlichen Souveräns zu akzeptieren. Bei Wahlentscheidungen ist diese Akzeptanz in der Bundesrepublik heute weitgehend gesichert. Wenn Art. 20 des Grundgesetzes bestimmt, dass die Staatsgewalt vom Volk in "Wahlen und Abstimmungen“ ausgeübt wird, dann ist derselbe Respekt vor dem Volk auch für direktdemokratisch getroffene Sachentscheidungen zu fordern.

Normativ lässt sich dies in zweifacher Hinsicht konkretisieren. Zum einen muss eine politische Schamfrist beachtet werden, innerhalb derer der Gesetzgeber auf ein volksbeschlossenes Gesetz nicht zugreifen darf. Am unbedenklichsten wäre es, damit bis zum Ablauf der Legislaturperiode zu warten und die geplante Änderung zum Gegenstand der Wahlauseinandersetzung zu machen. Ob sich ein Gesetz tatsächlich bewährt, kann man ohnehin erst nach einer gewissen Zeit feststellen. Gelingt es, die Mängel vor dem Wähler glaubhaft zu machen, birgt eine Korrektur oder Aufhebung aus legitimatorischer Sicht kein Problem.

Das zweite Kriterium bezieht sich auf das Maß der parlamentarischen Unterstützung, die die Änderung oder Aufhebung eines volksbeschlossenen Gesetzes erfährt. Wird eine solche Maßnahme von einer breiten Mehrheit, ja vielleicht sogar einstimmig beschlossen, ist zwar nicht ihre förmliche Legitimität, wohl aber ihre öffentliche Legitimationskraft zweifellos größer als bei einem einfachen Mehrheitsbeschluss, dem eine annähernd gleich starke Opposition widerspricht. Auch hier muss man sich aber vor Verallgemeinerungen hüten. Gerade in den unmittelbar machtrelevanten und politisch daher besonders heiklen Fragen der Wahlsystemgestaltung differieren die politischen Positionen erheblich. Zum Beispiel können hier kleine Parteien legitime Bestandsinteressen geltend machen,

(Hrsg.), Demokratie und Selbstverwaltung in Europa. Festschrift für Dian Schefold zum 65. Geburtstag, Baden-Baden 2001, S. 145 ff. (S. 157 ff). 
über die sich auch eine große Mehrheit nicht einfach hinwegsetzen darf. Darüber hinaus kann es Fragen geben, in denen eine breite Mehrheit oder sogar alle Parteien gegen die Mehrheitsmeinung der Bevölkerung stehen. Dies betrifft vor allem institutionelle Eigeninteressen, bei deren Verfolgung die Parteien nach Art eines Kartells funktionieren und deshalb dazu neigen, sich gegen Änderungen zu sperren. ${ }^{28}$ Neben dem Wahlrecht gilt das insbesondere für die Modalitäten der Politikfinanzierung sowie die Instrumente der direkten Demokratie.

Der Volksgesetzgebung gebührt in diesem Zusammenhang das Hauptinteresse, weil mit ihrer Hilfe institutionelle Reformen unmittelbar angestoßen und herbeigeführt werden können. ${ }^{29}$ Im günstigsten Fall entfaltet sich ihre Wirkung dabei präventiv, indem die Parteien angehalten werden, bestehende Regelungen von sich aus zu korrigieren. Im Hamburger Fall hat es an einem Konsens im Vorfeld leider gefehlt, mit dem sich der Weg über die Volksinitiative hätte abwenden lassen. Aus normativer Sicht führt folglich kein Weg daran vorbei, dass die Parteien den Volksbeschluss in all seinen Teilen hätten akzeptieren müssen.

\section{Konsens bei Wablrechtsreformen in der Bundesrepublik}

Die letztgenannte Feststellung wird auch dadurch gestützt, dass es in der Bundesrepublik eine verbreitete konsensuelle Praxis bei der Änderung von Wahlrechtsbestimmungen gibt. Angesichts der unmittelbaren Relevanz des Wahlsystems für die politischen Machtverhältnisse ist dies gut begründbar. Dass sich die Parteien auf einen Konsens leicht verständigen können, wenn es um die Sicherung ihrer gemeinsamen Eigeninteressen geht, wird niemanden verwundern. Ein solches Interesse könnte auch darin bestehen, das Aufkommen unliebsamer neuer Konkurrenz zu unterbinden. So hat man unlängst versucht, die Schwelle für eine Inanspruchnahme der staatlichen Parteienfinanzierung im Parteiengesetz heraufzusetzen, was vom Bundesverfassungsgericht allerdings für verfassungswidrig befunden wurde. ${ }^{30}$ Betrachtet man nur die etablierten Parteien, ist der Wahlrechtskonsens keineswegs selbstverständlich. Zum einen können die Wahlgesetze ja - wie gesehen - bereits mit einfachen Mehrheiten geändert werden. Zum anderen gibt es potenzielle

28 Vgl. Frank Decker, Parteiendemokratie im Wandel, in: ders. / Viola Neu (Hrsg.), Handbuch der deutschen Parteien, Wiesbaden 2007, S. 42 ff.

29 Dies betrifft die drei genannten Bereiche allerdings in unterschiedlichem Maße. Während die Regelungen der Parteienfinanzierung dem so genannten Finanztabu unterliegen und damit von der direkten Demokratie ganz ausgenommen sind, wird die Volksgesetzgebung auf der Ebene der Verfassung geregelt, deren Veränderung auch in einem direktdemokratischen Verfahren höheren Hürden unterliegt als die einfache Gesetzgebung. Die Ausführungsgesetze, die die Ausgestaltung der direkten Demokratie im Detail regeln und ihre Anwendbarkeit erschweren oder erleichtern, können demgegenüber auf der einfachgesetzlichen Ebene beschlossen werden. Letzteres gilt auch für das Wahlrecht, wo nur die allgemeinen Wahlgrundsätze auf der Verfassungsebene geregelt sind. Das Wahlsystem lässt sich deshalb sowohl über den Weg einer Volksinitiative als auch im regulären parlamentarischen Verfahren relativ leicht verändern.

30 BVerfG, 2 BvE 1/02 und 2 BvE 2/02 vom 26. Oktober 2004 = BVerfGE 111, S. 382 ff. Vgl. dazu auch Siegfried Jutzi, Zur Verfassungswidrigkeit des „Drei-Länder-Quorums“ bei der Parteienfinanzierung. Das Urteil des Bundesverfassungsgerichts vom 26. Oktober $2004-2$ BvE 1 und 2/02, in: ZParl, 36. Jg. (2005), H. 2, S. 375 ff. 
Interessenunterschiede zwischen großen und kleinen Parteien, die sich an der grundsätzlichen Systemalternative Mehrheits- oder Verhältniswahlrecht festmachen lassen. Die kleineren Parteien sind dabei zumindest theoretisch immer in der Gefahr, durch die Großen majorisiert und zum Verschwinden gebracht zu werden. Die Einführung eines Mehrheitswahlsystems lag während der ersten Großen Koalition 1966 bis 1969 in greifbarer Nähe. Nachdem sie an den Bedenken der SPD gescheitert war, wurde ein grundlegender Systemwechsel (der mit Sicherheit auch Veränderungen in den Ländern nach sich gezogen hätte) seither aber nicht mehr erwogen.

Alle Veränderungen des Wahlrechts, die nach Inkrafttreten des Bundeswahlgesetzes 1953 vorgenommen wurden, bewegten sich innerhalb der Grundstruktur eines Verhältniswahlsystems mit Fünf-Prozent-Sperrklausel und starren Listen. ${ }^{31}$ Nur die wenigsten dieser Änderungen wurden in einem breiten Konsens der kleinen und großen Parteien durchgesetzt. Die Fraktionsgrenzen wurden bei den Abstimmungen allerdings häufig überschritten. So fand das Bundeswahlgesetz 1953 nur bei der oppositionellen SPD und der FDP weitestgehende Unterstützung, während ein beträchtlicher Teil der Unionsabgeordneten und der Deutschen Partei dagegen votierten beziehungsweise sich der Stimme enthielten. Von wenigen Detailänderungen und notwendigen Anpassungen (etwa der Wahlkreiszuschneidung) abgesehen, wurde an diesem Gesetz bis heute nicht gerüttelt. Nach der deutschen Einheit geriet lediglich die so genannte Grundmandatsklausel kurzzeitig in die Diskussion, die der PDS 1994 den Einzug in den Bundestag sicherte, obwohl sie im Zweitstimmenergebnis unter fünf Prozent geblieben war. Obwohl Union und FDP diese Klausel mit ihrer Mehrheit hätten beseitigen können, verzichteten sie darauf. Dabei mögen Eigeninteressen eine Rolle gespielt haben (eine in Bayern unter die 50-Prozent-Marke absinkende CSU könnte irgendwann womöglich selbst auf die Regelung angewiesen sein); vor allem war es aber der Geruch eines manipulativen Eingriffs, der die Koalitionäre von dem Vorhaben Abstand nehmen ließ, denn eine Beseitigung der Klausel wäre von der Öffentlichkeit zu Recht als „lex PDS“ interpretiert worden.

Blickt man auf die Ebene der Länder, ergibt sich ein ähnliches Bild. Auch hier wurden Wahlrechtsreformen zum Teil konsensuell, zum Teil durch Mehrheitsbeschluss der Regierungsparteien herbeigeführt. Letzteres täuscht aber darüber hinweg, dass das Nein in vielen Fällen nicht auf einer grundsätzlichen Absage der Oppositionsparteien an die Reform beruhte, sondern darauf, dass man selbst noch weitergehende Reformvorstellungen hegte oder mit einer bestimmten Teilregelung nicht einverstanden war. Von den zahlreichen Beispielen sei hier nur die von CDU und FDP beschlossene Reform der hessischen Kommunalverfassung vom Dezember 1999 herausgegriffen, die SPD und Grüne nicht wegen der darin vorgesehenen offenen Listen ablehnten, sondern wegen der Wiederanhebung des Alters für das aktive Kommunalwahlrecht auf 18 Jahre.

Der Hamburger Fall ist hier deutlich anders gelagert. Seine Brisanz rührt daher, dass das Wahlrecht, das die CDU nun in seiner Substanz geändert hat, gegen den Willen der beiden großen Parteien vom Wahlvolk durchgesetzt worden war. Obwohl die von der CDU vorgenommenen Korrekturen in ihrer Tendenz der zurückhaltenderen Konkurrenzvorlage entsprechen, die 2004 auch von den Sozialdemokraten unterstützt worden

31 Eckhard Jesse, Reformvorschläge zur Änderung des Wahlrechts, in: APuZ, B 52 (2003), S. 3 11. Vgl. auch die Übersicht bei Peter Schindler, Datenhandbuch zur Geschichte des Deutschen 1949 bis 1999, Band 1, Baden-Baden 1999, S. $27-47$ 
war, beharrt die SPD nun auf dem Standpunkt, dass das von ihr eigentlich nicht gewollte Gesetz gelte und folglich auch angewendet werden müsse. ${ }^{32}$ Mit ihrer Revision des Wahlgesetzes stellt sich die Regierungsmehrheit demnach nicht nur gegen den Volksbeschluss, sondern auch gegen beide Oppositionsparteien im Parlament (SPD und Grüne). Dafür dürfte man in der Geschichte der Wahlrechtsreformen hierzulande kein vergleichbares Beispiel finden. Gewiss hat die Union Recht mit dem Hinweis, dass es ihr bei der Reform nicht darum gehe, sich selbst einen unbilligen Machtvorteil zu verschaffen. Dennoch erinnert ihr Vorgehen in mancherlei Hinsicht an die manipulative Praxis der Wahlrechtsreformen in anderen Ländern (etwa Italien oder Frankreich), von der sich die Bundesrepublik bisher immer wohltuend abgehoben hat.

\section{Zur Gefahr einer Entwertung der direkten Demokratie}

Dass sich die CDU der Problematik ihres Vorgehens durchaus bewusst zu sein scheint, lässt sich an zwei Dingen ablesen. Zum einen hat die Regierungspartei kein Interesse an einer breiten öffentlichen Debatte über die Wahlrechtsreform gezeigt, da sie zu Recht davon ausgehen musste, dass dies den Widerstand gegen das Vorhaben anheizen würde. Zum zweiten möchte sie das Wählerpublikum glauben machen, dass die von ihr geplanten Änderungen gar nicht so gravierend sind. ${ }^{33}$ Transparent sind diese Änderungen tatsächlich allein im Bereich der Zweitstimme, wo die Möglichkeit des Kumulierens und Panaschierens ganz entfällt und dem Wähler künftig nur noch eine Stimme zur Verfügung steht. Bei der Wahlkreisstimme bleibt die Grundstruktur des volksbeschlossenen Wahlgesetzes demgegenüber vorderhand unangetastet, so dass die weitreichenden Konsequenzen der Neuregelungen nicht unmittelbar erkennbar sind. Statt die Änderungen als solche auszuweisen und nach außen hin sichtbar zu machen, geht es der Regierungspartei hier wohl in erster Linie darum, die Radikalität ihres Entwurfs zu verschleiern. Dem Wähler soll ein Einfluss vorgegaukelt werden, den er bei Lichte betrachtet gar nicht (mehr) hat.

Dass das neuerliche Ignorieren eines Volksentscheides nicht geeignet ist, die Legitimität der Parteiendemokratie zu stärken, liegt auf der Hand. Der respektlose Umgang mit den Bürgern dürfte im Gegenteil eine Entwicklung befördern, die schon heute zu einem besorgniserregenden Anstieg der Politikverdrossenheit geführt hat. Setzen sich die Parteien über Volksbeschlüsse einfach hinweg, wird das dem Ressentiment Vorschub leisten, man sei der "parteipolitischen Klasse“ ohnehin auf Gedeih und Verderb ausgeliefert. Die Wähler könnten ein solches Verhalten als schiere Machtarroganz empfinden. Dies würde auch zu einer Entwertung der direktdemokratischen Institutionen selbst führen. Wenn die Bürger auf die Gültigkeit ihrer Entscheidungen nicht mehr vertrauen können, werden sie sich entweder resignierend zurückziehen; oder sie suchen sich andere Kanäle des Widerspruchs und Protests wie etwa die Unterstützung populistischer oder gar extremis-

32 Vgl. dazu die Debattenbeiträge bei der 1. und 2. Lesung des Gesetzes am 27. September und 11. Oktober 2006 in der Bürgerschaft: Bürgerschaft der Freien und Hansestadt Hamburg, Plenarprotokolle 18/64 und 18/65.

33 Vgl. Bürgerschaft der Freien und Hansestadt Hamburg, Protokoll der öffentlichen Sitzung des Verfassungsausschusses vom 4. Juli 2006 (Nr. 18/23). 
tischer Parteien, die mit Blick auf die Systemstabilität sehr viel problematischer sind. Dafür liefert gerade Hamburg einiges Anschauungsmaterial. So verdankten sich nicht nur die Entstehung und der Erfolg der Statt-Partei im Jahre 1993 einer „Demokratiekrise“. Auch die Stimmenzuwächse der rechtsextremen Parteien und der Sensationserfolg des Rechtspopulisten Ronald Schill im Jahre 2001 hatten ihre Ursache in einer tiefgehenden Unzufriedenheit mit den etablierten politischen Kräften. ${ }^{34}$ Gewiss wurde dieses Potenzial 2004 von den großen Parteien zum Teil wieder integriert. Doch könnten schon bald neue Akteure auf den Plan gerufen werden, die sich den mangelnden Respekt der Politiker vor den demokratischen Institutionen als Thema zunutze machen.

Gemessen an der Situation des Jahres 1993 bleibt das Interesse der Hamburger Bevölkerung am Demokratiethema bislang noch gering. ${ }^{35}$ Andere Fragen rangieren in der öffentlichen Aufmerksamkeit höher, weshalb die CDU hoffen mag, der Wähler werde ihr das demokratiewidrige Verhalten nachsehen und sie bei den nächsten Bürgerschaftswahlen ohne größere Abstrafung davonkommen lassen. Es ist ein generelles Problem der parlamentarisch-repräsentativen Demokratie, dass die Politiker überwiegend in kurzfristigen Kategorien des Machtgewinns oder -verlusts denken und die längerfristigen Folgen ihres Tuns vernachlässigen. Bezieht man diese mit ein, ist das Vorgehen der Union in doppelter Hinsicht fragwürdig. Zum einen nimmt die Partei die Beschädigung der demokratischen Institutionen offenbar in Kauf beziehungsweise ordnet sie diese ihrem Interesse unter, die Kontrolle über die Rekrutierung der Abgeordneten zu bewahren oder zurückzugewinnen. Zum anderen verkennt sie, dass die korrigierenden Elemente der Volksgesetzgebung einer Regierungspartei durchaus helfen können, die eigene Mehrheit zu erhalten. Der verlorene Volksentscheid über den Krankenhausverkauf, der im Februar 2004 zeitgleich mit der Bürgerschaftswahl stattfand, liefert dafür ein gutes Beispiel. Wäre diese Frage nicht separat entschieden worden, sondern im Kontext der allgemeinen Wahlauseinandersetzung verblieben, hätte die rot-grüne Opposition auf zusätzliche Stimmen hoffen können und die Union ihre absolute Mehrheit womöglich verfehlt. ${ }^{36}$ So aber haben sich die Wähler bei der Bürgerschaftswahl ganz vom Persönlichkeitsfaktor der Spitzenkandidaten leiten lassen, wo die CDU mit Ole von Beust deutlich im Vorteil war. ${ }^{37}$

Eine andere, sehr viel grundsätzlichere Frage betrifft die generelle Ausgestaltung der Direktdemokratie in den Ländern. Diese hat sich bisher stets auf die vermeintlich fort-

34 Vgl. dazu auch Julia von Blumenthal, Die Schill-Partei und ihr Einfluss auf das Regieren in Hamburg, in: ZParl, 35. Jg. (2004), H. 2, S. 271 ff.

35 Bei der öffentlichen Anhörung zur Wahlrechtsänderung, die von der CDU-Mehrheit kurzfristig und zeitgleich mit der Fernsehübertragung der Fußball-WM angesetzt wurde, war am 16. Juni 2006 nur ein (!) interessierter Bürger anwesend. Die Initiatoren des volksbeschlossenen Wahlrechts hatten ihre Teilnahme mit dem Hinweis abgesagt, es handele sich um eine „scheindemokratische Alibiveranstaltung".

36 Darauf weist unter anderem das - entgegen früheren Gepflogenheiten - abweichende Ergebnis der Wahlen zu den sieben Bezirksversammlungen hin, bei denen die Christdemokraten schlechter und SPD und Grüne besser abschnitten als bei der Bürgerschaftswahl.

37 Beim Referendum und bei der Vetoinitiative haben die Bürger also „lediglich“ die Möglichkeit, über ein parlamentarisch zustande gekommenes Gesetz nochmals abzustimmen (und dies gegebenenfalls zu verwerfen), während sie im Rahmen der Volksgesetzgebung selbst legislativ tätig werden und als Beschlussorgan fungieren. Vgl. Frank Decker, Direkte Demokratie auf hanseatisch. Warum das parlamentarische Regierungssystem im Stadtstaat überholt ist, in: MUT. Forum für Kultur, Politik und Geschichte Nr. 442 (Juni 2004), S. 14 - 17. 
schrittlichste Variante der Volksgesetzgebung kapriziert, wofür es in Westeuropa - von der Schweiz abgesehen - kein vergleichbares Beispiel gibt. Das Problem der auch in Hamburg praktizierten Trias von Volksinitiative, Volksbegehren und Volksentscheid liegt darin, dass die Bürger dabei in letzter Konsequenz an die Stelle - und nicht nur an die Seite - des parlamentarischen Gesetzgebers treten können. Deshalb ist diese Form direkter Demokratie, was die Systemverträglichkeit angeht, prekärer als andere wie eine Vetoinitiative oder solche Referenden, denen der parlamentarische Beschluss bereits vorausgeht. ${ }^{38}$ Dies macht sich in doppelter Hinsicht negativ bemerkbar: Zum einen führt es dazu, dass der Verfassungsgeber die Anwendbarkeit der direktdemokratischen Instrumente vorsorglich beschränken muss, indem er bestimmte Ausschlussgegenstände festlegt und Quoren vorschreibt. Zum anderen verstärkt es die Konflikte, falls es den Initiatoren doch einmal gelingen sollte, die Hürden zu überspringen und sich gegen den Willen des parlamentarischen Gesetzgebers zu stellen. Der Hamburger Fall könnte so gesehen auch als Hinweis genommen werden, dass sich der vermeintliche Königsweg der direkten Demokratie in Wahrheit als Holzweg entpuppt. Die Frage lautet also, ob die Verfassungsgeber in der Bundesrepublik gut beraten sind, sich bei der Ausgestaltung der direkten Demokratie ausschließlich auf die Volksgesetzgebung zu konzentrieren. Sie müsste dringend beantwortet werden, wenn plebiszitäre Verfahren auch ins Grundgesetz eingeführt werden sollen. Eine umstandslose Übertragung des Modells, wie es heute in den Ländern besteht, scheint hier wenig ratsam. Dies gilt um so mehr, als auf der Bundesebene noch weitere schwierige Probleme zu lösen wären (etwa die Frage nach einer angemessenen Beteiligung der Zweiten Kammer ${ }^{39}$ ), die in der rechts- und politikwissenschaftlichen Forschung bislang kaum angesprochen worden sind. ${ }^{40}$ Diese Probleme würden bei einer „bescheideneren" Variante der Direktdemokratie entfallen.

38 Frank Decker, Die Systemverträglichkeit der direkten Demokratie. Dargestellt an der Diskussion um die Einführung von plebiszitären Elementen in das Grundgesetz, in: ZPol, 15. Jg. (2005), H. 4, S. 1103 - 1147.

39 Dazu jetzt Denise Estel, Bundesstaatsprinzip und direkte Demokratie im Grundgesetz, BadenBaden 2006 sowie Sebastian Blasche, Die grundsätzliche Mitwirkung der Länder bei der Gesetzgebung. Eine verfassungsdogmatische Untersuchung zu Art. 79 Abs. 3, 2. Var. GG vor dem Hintergrund einer möglichen Einführung von Volksgesetzgebung in das Grundgesetz, BadenBaden 2006.

40 Vgl. Uwe Leonardy, Plebiszit und Bundesstaat: Eine Rezension mit Ergänzungen zur Diskussion, in: ZParl, 23. Jg. (1992), H. 1, S. 163 ff. 